\title{
Molecular diagnostics for precision medicine in breast cancer treatment: what does the future hold?
}

\author{
Shigehira Saji $^{1} \cdot$ Reiko Kimura-Tsuchiya $^{1} \cdot$ Eisaku Sasaki $^{1}$
}

Published online: 3 November 2015

(C) The Japanese Breast Cancer Society 2015

Personalized medicine or precision medicine is one of the most interesting topics in current clinical cancer research. When compared to other types of solid cancer, breast cancer has been utilized sub-personalized (subtyping) treatment strategy for a very long time. In the 1980s, the presence of estrogen receptor (ER) was evaluated by a dextran-coated charcoal assay (ligand binding assay) and endocrine therapy was administered to specific patients with ER-positive breast cancer [1]. LHRH analog for premenopausal patients is another subtyping approach. However, recent technological advances in multi-gene assay and whole genome sequencing have made it possible to divide breast cancer subtyping into more detailed categories. In addition, classical RT-PCR technique for mRNA expression analysis was developed to digital PCR assay with high sensitivity. Based on these technological advances, we are trying to administer the right drug at the right time to the right patient. The development of molecular diagnostic tools is a key element for realizing precision and personalized medicine in clinical practice. These tools should be industrialized and approved by a regulatory agency.

This special feature issue of Breast Cancer includes five review articles related to molecular diagnostics. Figure 1 shows the application of each molecular diagnostic in clinical breast cancer time course. For instance, multi-gene classifiers are used for prediction of risk of recurrence, and companion diagnostics are applied for choosing a specific

Shigehira Saji

ssaji@fmu.ac.jp

1 Department of Medical Oncology, Fukushima Medical University, School of Medicine, 1 Hikarigaoka, Fukushima, Fukushima 960-1295, Japan drug for each patient. Sato et al. cover the basic knowledge of "genomic tumor evolution". Recent progress of comprehensive genome-wide analysis has revealed information regarding genomic mutations and rearrangements in each individual tumor [2]; however, during the process of tumor invasion, metastasis and treatment exposure, cancer genomes are dynamically changing and evolving. At this moment, no established technique exists for tracking this genomic tumor evolution in the living tumor of a patient, although liquid biopsy to detect circulating tumor cells and circulating cell-free DNA could be a promising approach.

Naoi et al. reported multi-gene classifiers for predicting recurrence risk for early breast cancer patients [3]. In contrast to conventional biomarkers such as ER, PgR and HER2, multi-gene assay evaluates multiple target mRNA expressions at one time, providing a significant amount of information for treatment decision. Although many multigene assays have been developed to date, most success case is the Oncotype DX assay which calculates the recurrence score (RS) based on the expression of 21 genes using RNA extracted from FFPE tumor tissues. Very recently, the first survival data of the TAILORx study with Oncotype DX has been reported [4]. Early breast cancer patients with ERpositive HER2-negative node-negative tumors and those whose tumors were classified as low RS (equal or less than 10) showed remarkable good prognosis without adjuvant chemotherapy. This is the first prospective evidence for treatment decision to have been made using multi-gene assay.

Tazawa reported on the development of companion diagnostics for indication of molecular targeting drugs [5]. Though many molecular targeting drugs for breast cancer have been developed to date, the most scene-changing drug has been trastuzumab for HER2-positive tumor. Trastuzumab was developed through clinical trials that 


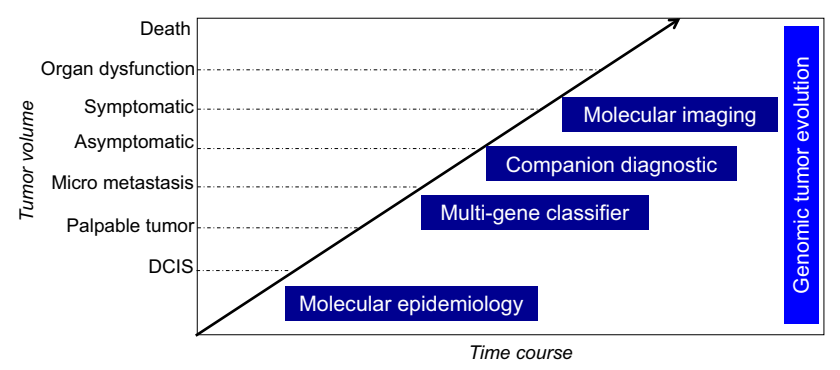

Fig. 1 Application of molecular diagnostic tools in clinical breast cancer time course. $Y$ axis indicates tumor volume, and $X$ axis indicates time course of disease. DCIS ductal carcinoma in situ

restricted enrolled patients, whose tumor overexpressed HER2 protein, leading to a remarkable overall response rate as well as a significant prolongation in overall survival. In this process, a companion diagnostic tool named Herceptest was simultaneously developed. Although the same approach has been tried with other drugs such as bevacizumab and everolimus, they failed to find predictive biomarkers of response and could not establish companion diagnostic, resulting in non-significant benefits in overall survival. Table 1 shows the molecular targeting drugs for ER-positive breast cancer, currently being developed in phase III trials [6]. All of these drugs are used alongside endocrine therapy; however, no specific predictive biomarkers have been found during the developmental process. Due to high cost of new molecular targeting drugs and very competitive developmental situations aiming at the same targets, such as CDK4/6 inhibitor, a companion assay for prediction of the benefits of each drug is necessary. Regulatory agencies' problems of approving laboratory-developed test are also discussed in this paper.

Kurihara et al. reported molecular imaging using positron emission tomography (PET) for evaluating in vivo biomarkers and drug response [7]. Molecular imaging can visualize the biological process at molecular and cellular levels in vivo, using specific tracers for molecular targets. In clinical practice, molecular imaging could be used for the detection of cancer in vivo, defining the characteristics of tumor subtypes by using molecular tags. Moreover, molecular imaging could be used for the evaluation of treatment response in earlier time course during treatment. It is likely that hormone receptor PET imaging would be able to provide the most useful information, if it could be developed to be a valid diagnostic test. The PET tracer $16 \alpha^{-18} \mathrm{~F}$-fluoro-17 $\beta$ estradiol can bind to ER in a patient's tumor, and monitor receptor expression status in a non-invasive and repeated manner. The basic treatment strategy of ERpositive metastatic breast cancer is sequential endocrine therapy; however, response becomes weaker in later treatment sequences. If the ER expression in later periods could be confirmed, we would be able to try the

Table 1 Molecular targeting drugs and clinical trial settings for ER-positive HER2-negative breast cancer

\begin{tabular}{|c|c|c|c|c|c|}
\hline Inhibitor & & Trial $(n)$ & Phase & Endocrine & Patient group \\
\hline HDAC & Entinostat & ECOG-ACRIN $(n=600)$ & III & Exemestane & Postmeno, 2nd line \\
\hline \multirow[t]{9}{*}{ CDK4/6 } & \multirow{4}{*}{$\begin{array}{l}\text { Palbociclib (PD- } \\
\text { 0332991) }\end{array}$} & PALOMA-2 $(n=650)$ & III & Letrozole & Postmeno, 1st line \\
\hline & & PALOMA-3 $(n=417)$ & III & Fulvestrant & $\begin{array}{l}\text { Post and premeno with LHRHa, 2nd } \\
\text { line }\end{array}$ \\
\hline & & PALOMA-4 $(n=330)$ & III & Letrozole & Asian postmeno, 1st line \\
\hline & & PEARL $(n=348)$ & III & $\begin{array}{l}\text { Exemestane vs. } \\
\text { capecitabine }\end{array}$ & Postmeno, 2nd line \\
\hline & \multirow[t]{3}{*}{ Ribociclib (LEE011) } & $\begin{array}{l}\text { MONALEESA-2 } \\
\quad(n=650)\end{array}$ & III & Letrozole & Postmeno, 1st line \\
\hline & & $\begin{array}{l}\text { MONALEESA-3 } \\
\quad(n=660)\end{array}$ & III & Fulvestrant & Postmeno, 1st, 2nd line \\
\hline & & $\begin{array}{l}\text { MONALEESA-7 } \\
(n=660)\end{array}$ & III & $\begin{array}{l}\text { Tamoxifen/ns-AI } \\
+ \text { LHRHa }\end{array}$ & Premeno, 1st line \\
\hline & \multirow[t]{2}{*}{$\begin{array}{l}\text { Abemaciclib } \\
\text { (LY2835219) }\end{array}$} & MONARCH $2(n=550)$ & III & Fulvestrant & $\begin{array}{l}\text { Post and premeno with LHRHa, 2nd } \\
\text { line }\end{array}$ \\
\hline & & MONARCH $3(n=450)$ & III & ns-AI & Postmeno 1st line \\
\hline \multirow[t]{2}{*}{ mTOR } & \multirow[t]{2}{*}{ Everolimus } & BOLERO-2 $(n=724)$ & III & Exemestane & Postmeno 2nd line \\
\hline & & SWOG $1222(n=825)$ & III & Fulvestrant + anastrozole & Postmeno, stage-IV 1st line \\
\hline $\begin{array}{l}\text { Pan- } \\
\text { PI3K }\end{array}$ & Buparlisib (BKM120) & BELLE-2 $(n=842)$ & III & Fulvestrant & Postmeno 2nd line \\
\hline
\end{tabular}

$n s-A I$ non steroidal aromatase inhibitor, LHRHa LH-RH analog, postmeno postmenopausal, premeno premenopausal 
next endocrine therapy and avoid early use of chemotherapy [8]. In addition to ER, HER2 expression in vivo is also visualized by HER 2 monoclonal antibody labeled with radioactive molecules. In general, conventional fluorodeoxyglucose (FDG) PET imaging is used for the detection of cancer. Ellis et al. used FDG-PET CT for monitoring metabolic flare after estradiol tablet treatment in vivo [9]. Estrogen such as diethylstilbestrol is an established endocrine therapy for late-stage MBC patients. They reported that such metabolic flare was a highly significant predictor of response to estradiol treatment.

A study by Ito and Matsuo reported the history and recent topics of molecular epidemiology for personalized cancer prevention [10]. Cancer is generally thought to be a multi-factorial disease consistent with environmental factors and genetic factors. Lung cancer, for instance, although smoking history is the strongest initiation factor, not all heavy smokers get lung cancer. Molecular epidemiology provides an answer to this question by "geneenvironment interaction". Recent advances in genotyping technology make it possible to conduct genome-wide association studies (GWAS) for single nucleotide polymorphisms. The "Gail model" is the most commonly used prediction tool for breast cancer, incorporating personal medical, reproductive and familial histories. Furthermore, recent GWASs have identified multiple genetic variants associated with breast cancer risk [11]. Efforts to add these variants to existing risk-assessment models are currently underway. It is our belief that, in the near future, we will see breast cancer risk prediction using genetic and environmental risk factors, enabling personalized precision prevention.

\section{References}

1. Palmieri C, Saji S, Gustafsson JA, Coombes RC. False negatives in oestrogen-receptor assay. Lancet. 2000;356:944-45.

2. Sato F, Saji S, Toi M. Genomic tumor evolution of breast cancer. Breast Cancer. 2015. doi:10.1007/s12282-015-0617-8.

3. Naoi Y, Noguchi S. Multi-gene classifiers for prediction of recurrence in breast cancer patients. Breast Cancer. 2015. doi:10. 1007/s12282-015-0596-9.

4. Sparano JA, Gray RJ, Makower DF, Pritchard KI, Albain KS, Hayes DF, et al. Prospective validation of a 21-gene expression assay in breast cancer. N Engl J Med. 2015. doi:10.1056/ NEJMoa1510764.

5. Tazawa Y. Perspective for the development of companion diagnostics and regulatory landscape to encourage personalized medicine in Japan. Breast Cancer. 2015. doi:10.1007/s12282015-0586-y.

6. Saji S, Kimura-Tsuchiya R. Combination of molecular-targeted drugs with endocrine therapy for hormone-resistant breast cancer. Int J Clin Oncol. 2015;20:268-72.

7. Kurihara H, Shimizu C, Miyakita Y, Yoshida M, Hamada A, Kanayama Y, et al. Molecular imaging using PET for breast cancer. Breast Cancer. 2015. doi:10.1007/s12282-015-0613-z.

8. van Kruchten M, de Vries EG, Brown M, de Vries EF, Glaudemans AW, Dierckx RA, et al. PET imaging of oestrogen receptors in patients with breast cancer. Lancet Oncol. 2013;14:e465-75.

9. Ellis MJ, Gao F, Dehdashti F, Jeffe DB, Marcom PK, Carey LA, et al. Lower-dose vs high-dose oral estradiol therapy of hormone receptor-positive, aromatase inhibitor-resistant advanced breast cancer: a phase 2 randomized study. JAMA. 2009;302:774-80.

10. Ito $\mathrm{H}$, Matsuo $\mathrm{K}$. Molecular epidemiology, and possible realworld applications in breast cancer. Breast Cancer 2015;2-7. doi: 10.1007/s12282-015-0609-8.

11. Wacholder S, Hartge P, Prentice R, Garcia-Closas M, Feigelson HS, Diver WR, et al. Performance of common genetic variants in breast-cancer risk models. N Engl J Med. 2010;362:986-93. 